\title{
Cancer Patient Management during COVID-19 Pandemic: An Audit of a Single-Surgeon Unit in a COVID-Hotspot
}

\author{
Laleh Busheri ${ }^{1} \quad$ Smeeta Nare ${ }^{1}$ Rituja Banale ${ }^{1}$ Ashwini Bapat ${ }^{1} \quad$ Moushumi Nagarkar ${ }^{1}$ Deepali Vetale \\ Rebecca Joseph ${ }^{1}$ Swapnil Suryawanshi ${ }^{1}$ Rashmi Alhat ${ }^{1}$ George Thomas ${ }^{1}$ Ruth Navgire ${ }^{1}$ \\ Danish Ansari ${ }^{1}$ Rohini Unde ${ }^{1}$ Shahin Shaikh ${ }^{1}$ Nutan Gangurde ${ }^{1}$ Santosh Dixit ${ }^{1,2}$ \\ Beenu Varghese ${ }^{1}$ Chetan Deshmukh¹ Devaki A. Kelkar ${ }^{1,2}$ Madhura Kulkarni ${ }^{1,2}$ \\ Chaitanyanand B. Koppiker ${ }^{1,2}$
}

\author{
${ }^{1}$ Orchid Breast Health Clinic and Prashanti Cancer Care Mission, \\ Pune, Maharashtra, India \\ ${ }^{2}$ Center for Translational Cancer Research, A Joint Initiative between \\ IISER Pune and Prashanti Cancer Care Mission, Pune, Maharashtra, \\ India
}

\section{South Asian J Cancer 2021;10:39-41.}

\begin{abstract}
Address for correspondence Madhura Kulkarni, PhD, Centre for Translational Cancer Research and Prashanti Cancer Care Mission, 1-2 Kapil Vastu, Senapati Bapat Road, Pune 411016, India (e-mail: madhura.kulkarni@prashanticancercare.org).
\end{abstract}

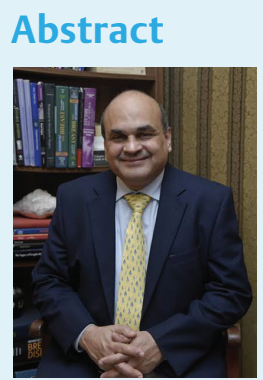

Dr. Madhura Kulkarni
The report evaluates the effect of coronavirus disease (COVID-19) pandemic on breast cancer treatment and management at a single-surgeon cancer care unit in one of the hotspots of COVID-19 in India. In response to the pandemic, the adjustments were made in the clinical practice to accommodate social distancing. Patient consultations were done over phone call or in-clinic visit with prior appointment to reduce the risk of exposure to COVID-19. Total number of patients that were treated at the clinic and the essential surgeries performed during the pandemic phases are summarized in the report. The methodology adopted here for care and management of the cancer patients can serve as a guiding principle for cancer care units in the country.

Keywords

breast cancer, cancer treatment and management, COVID-19 pandemic

\section{Introduction}

The pandemic with coronavirus disease (COVID-19) has affected health care system, especially changing the routine practices meant for chronic illnesses such as cancer. Treatment delivery for cancer patients faced unusual challenges during the pandemic to avoid and reduce the risk of exposure to COVID-19 and to comply with the government regulations. The hospitals and clinics across the country have raised to the occasion with careful planning and adjustments to minimize the risk of exposure while continuing with diagnosis and treatment of cancer patients with limited resources. ${ }^{1-3}$

DOI https://doi.org/10.1055/s-0041-1731578 ISSN 2278-330X

How to cite this article: Busheri L, Nare S, Banale R, et al. Cancer Patient Management during COVID-19 Pandemic: An Audit of a Single-Surgeon Unit in a COVID-Hotspot South Asian J Cancer 2021;10(1):39-31.
This report presents the efforts of continued cancer treatment through the pandemic at a single surgeon, breast cancer care unit in one of the hotspot cities of India, with limited international and national guidelines. ${ }^{3}$

\section{Methods}

\section{Measures Taken to Reduce COVID Exposure to the \\ Patients}

COVID-19 was detected in India in mid-March and the government declared complete lockdown starting from March 25 till May 31, 2020. From June 1, 2020, unlock phases were

(C) 2021. MedIntel Services Pvt Ltd.

This is an open access article published by Thieme under the terms of the Creative Commons Attribution-NonDerivative-NonCommercial-License, permitting copying and reproduction so long as the original work is given appropriate credit. Contents may not be used for commercial purposes, or adapted, remixed, transformed or built upon. (https://creativecommons.org/licenses/by-nc-nd/4.0/).

Thieme Medical and Scientific Publishers Private Ltd A-12, Second Floor, Sector -2, NOIDA -201301, India 
introduced through the year maintaining social distancing norms.

The clinic invested first 2 weeks to gather all the necessary supply of personal protective equipment (PPEs), disinfectants, shields, and hand sanitizers for the staff on board. During the lockdown number of clinic admissions fell to nil, and elective surgeries were purposefully curtailed in the clinic to avoid risk of exposure. During the phase 1 of the lockdown, the surgeon on board operated three cases at Jehangir Hospital, Pune, Maharashtra, India, which were critical from the cancer treatment point of view. The rest of the patients were followed up over phone call.

With effect from May 1, 2020, outpatient department (OPD) was opened for emergency cases only, between 12 to 4 p.m. Indian Standard Time; with restricted entry for patients with cough, cold and sore throat, or fever. During the unlock phase, the clinic began to operate twice a week, with patients for postoperative and annual follow-ups, or urgent cases. A few cases with newly diagnosed disease were also consulted for. From October 1, 2020, the OPD reopened to full working hours for all clinical staff. All cases and follow-ups resumed for new/annual and postoperative care.

All the operations and visits were conducted with staff in complete PPE and following the social distancing norms with utmost sanitization practices.

\section{Data Retrieval and Analysis}

At the clinic, patients' record and information are logged in PALASH software for consultation and treatment details. The number patients per month for OPD visits, in-patient treatment, and their pharmacy-related activities and surgery details were retrieved from PALASH to an Excel sheet. The surgeries done per month during the pandemic are plotted for comparison with the pre-COVID era (2019).

\section{Results}

With measures to curb the exposure to COVID-19, the number of patients visiting the clinic has varied through the lockdown phases. In-person consultations in the month of April were zero owing to curb the spread with stringent government regulations of phase 1 . The number of consultations started going up from the month of May 2020. The highest number of consultations were seen in the month of September $2020(n=220)$ as the regulations were relaxed. The number of surgeries performed for breast cancer treatment during the lockdown was only of essential nature. COVID test was performed prior to the operative procedure. Only COVID negative patients were operated.

\section{Impact of the Pandemic on the Surgery Treatment}

The first phase of the lockdown in April had witnessed three surgeries that were planned in advance and were essential part of the treatment of the patients. There was a slow rise in the number of surgeries, which reached the peak of 23 in June 2020. The stringent regulations, owing to increasing COVID-19 cases in mid-July resulted in a decline in the number of surgeries in the month of July and August ( - Fig. 1 ).
The number reached 21 in the month of October 2020 as the unlock phases were introduced. Even with such stringent measures, the clinic was able to offer care and treatment which where most essential. During the pandemic, the clinic was able to achieve capacity close to the pre-COVID era ( - Fig. 1).

\section{COVID Positive Breast Cancer Patients}

Through the pandemic, one breast cancer patient was tested positive in preoperative COVID test in September 2020. The patient was operated once COVID negative, in October 2020. Two cases became positive through family contact during their course of postoperative chemotherapy and were asymptomatic. The therapy was kept on hold during their home isolation and was resumed once the patients became COVID negative.

\section{Discussion/Conclusion}

As the world was adjusting to the COVID-19 pandemic, India also rose to curtail the spread and to protect the individuals with phased lockdown. This meant significant adjustments were to be made within medical practices especially for chronic diseases with long-term treatment and follow-up such as breast cancer. Various public and private medical practices were adjusted to accommodate social distancing, avoiding physical presence as much possible while maintaining patient's clinical interests as a priority. ${ }^{4}$

Ours is one of the highly acclaimed cancer clinics with state-of-the-art treatment for breast cancer with specialty in oncoplastic surgery. Along with de novo diagnosis, our center gets referrals from around the state for surgical treatment. In line with the new normal life during the pandemic, our clinic incorporated social distancing and PPE early on. The patient interaction in person was reduced to minimum and with prior appointment to maintain minimum occupancy with optimal social distancing. With these efforts, the clinic was able to continue with the optimal care and treatment for the breast cancer patients despite the pandemic situation.

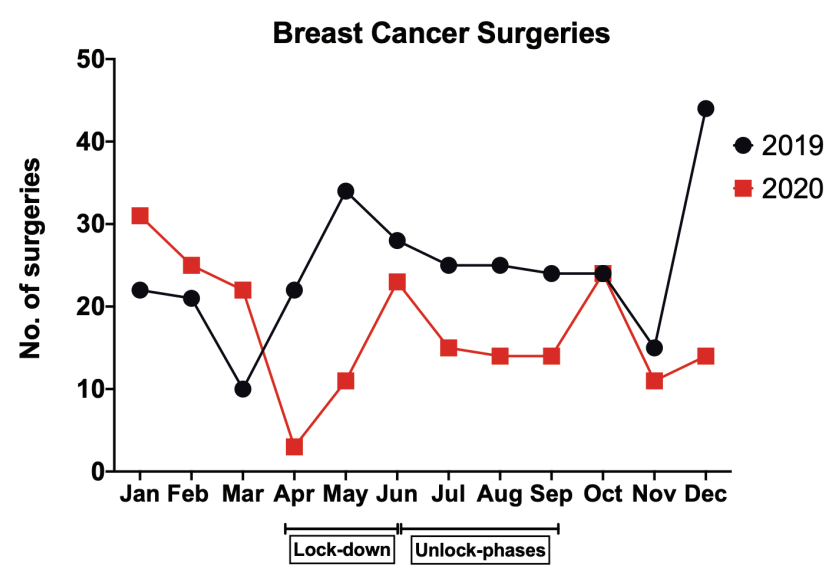

Fig. 1 Surgeries performed in pre-coronavirus disease (COVID) and during COVID times. Number of breast cancer surgeries that were performed by the surgeon on board per month compared for the year 2019 (pre-COVID) and 2020 (during COVID pandemic). 
The methods presented here in the report can be a reference to follow optimal standard of care for cancer patients during the pandemic.

\section{Conflict of Interest}

None declared.

\section{Acknowledgments}

M.K. would like to acknowledge DBT- Ramalingaswami "re-entry" fellowship awarded by DBT-India. Research grant to Dr. Koppiker is supported by Bajaj Auto Ltd.

\section{References}

1 Parasnis A, Kelkar D, Khaladkar B, et al. Continuing cancer treatment in a "COVID HOTSPOT" in India: are we overestimating the risks? Indian J Med Paediatr Oncol 2020;41:634-639

2 Deshmukh S, Naik S, Zade B, et al. Impact of the pandemic on cancer care: lessons learnt from a rural cancer center in the first 3 months. J Surg Oncol 2020;122(5):831-838

3 Pramesh CS, Badwe RA. Cancer management in India during Covid-19. N Engl J Med 2020;382(20):e61

4 Singh HK, Patil V, Chaitanya G, Nair D. Preparedness of the cancer hospitals and changes in oncosurgical practices during COVID-19 pandemic in India: a cross-sectional study. J Surg Oncol 2020;122(7):1276-1287 